\title{
INFLUENCE DE DIFFERENTES DOSES D'HORMONE GONADOTROPE SÉRIQUE (PMSG) INJECTÉES EN PHASE FOLLICULAIRE SUR LA DURÉE DU GYCLE CESTRIEN CHEZ LES BOVINS
}

\author{
P. MAUlÉon, J.-C. MARIANA, D. CHUPIN et Aline SOLARI \\ avec la collaboration technique de Y. DE FONTAUBert \\ Institut national de la Recherche agronomique \\ Laboratoire de Physiologie de la Reproduction, 37 - Nouzilly, France \\ Station centrale de Physiologie animale, 78 -Jouy-en-Josas, France
}

Chez la Vache, l'injection d'hormones gonadotropes à n'importe quel moment du cycle et même pendant la gestation provoque l'ovulation (ZAVADovski et al., I935). Mais lorsque cette injection est effectuée en phase progestative, les vaches extériorisent rarement un comportement d'œestrus (HAMmond et BHATTACHARYa, r944) et les œufs pondus sont peu fécondables (CASIDA et al., I940 ; RowSOn, I95I ; WILLETT et al., I952).

Par contre, lorsque l'injection de PMSG est faite vers la fin du cycle, les vaches viennent généralement en ostrus (HAMmond, BhatTaChaRya, I944; Gordon et al., I962; Hafez et al., I965; Scanlon et al., I968). La fécondabilité des œufs pondus est élevée bien que le pourcentage de survie des œufs superovulés et fécondés varie en fonction de l'intervalle entre l'injection de PMSG et l'œstrus (IC) puisque les œufs dégénèrent lorsque cet intervalle est supérieur à 5 jours (LAMMING et RowSON, I952). La durée de cet intervalle influe également sur la variabilité du taux de superovulation obtenu (HAFEZ et al., I965; SCANLON et al., I968).

Or l'injection de PMSG modifierait légèrement la durée moyenne du cycle et rendrait ainsi difficile le calcul de l'intervalle IC, elle entrainerait également des anomalies d'œstrus (ovulations silencieuses) plus fréquentes que normalement (HAFEz et al., I965). Cependant il n'a pas toujours été tenu compte de la variabilité individuelle de la durée du cycle oestrien (Olds et SEATH, I95I). Aussi avons-nous repris cette étude et recherché si la durée du cycle œstrien était perturbée par l'injection de PMSG effectuée en phase folliculaire; nous avons effectué au préalable une correction du moment d'injection de PMSG d'après la durée moyenne des cycles œstriens antérieurs de chaque individu. 


\section{MATÉ,RIEL, E'T MÉTHODES}

Cette étude a porté sur 323 vaches de race Française Frisonne Pie Noire (expériences A I et B IV), primipares, en lactation et traites. Les cycles ostriens ont été observés depuis la mise bas et les animaux ont reçu le traitement PMSG entre 85 jours et $\mathbf{I}_{4}$ o jours après la mise bas. Les observations ont été faites également sur les 62 vaches de race Charolaise (expérience A II) primipares et allaitant leurs veaux; ces animaux sont entrés en expérience environ Ioo jours après la mise bas. L'injection de PMSG a toujours été effectuée de 8 h 30 à 9 h 30 , quatre jours avant la date présumée de l'œstrus; cette dernière a été calculée en utilisant la durée moyenne de trois cycles oestriens.

Dans le dépouillement concernant la venue en astrus, il n'a pas été tenu compte de l'injection ou non de l'hormone chorionique puisque celle-ci n'est faite qu'après le début observé de l'oestrus. Un soin particulier a été apporté à la détection de celui-ci de façon à obtenir avec précision l'heure de début et de fin de chalcurs. Au cours de l'expérience réalisée au C. N. R. Z. de Jouy-en-Josas le comportement vis-à-vis de deux mâles vasectomisés était noté chaque matin et chaque soir. Les vaches par groupe de dix séjournaient un quart d'heure dans un parc où le taureau était présent; la position des vaches par rapport au mâlc attaché était notée au bout de 3 minutes et les activités de chevauchement relevées, cet ensemble de critères permettant de définir le début de l'œestrus.

Au cours des expériences A II et B IV, réalisées au C. R. V. Z. de Nouzilly la technique d'utilisation du taureau vasectomisé a dû être simplifiée, mais la fréquence de passage est restée la même. Le mâle est lâché au milieu du groupe de I 5 à 20 vaches. Les réactions d'immobilisation des femelles constituent le seul critère d'œestrus mais l'activité de recherche du mâle par les femelles aide à orienter le taureau vers la découverte des femelles en cestrus; celles-ci sont sorties du lot aussitôt que le test d'immobilité est obtenu. Nous avons estimé que la précision dans la détermination du début de l'cestrus était de \pm 3 heures. Elle est beaucoup plus faible avec ces méthodes en ce qui concerne la fin de l'cestrus, aussi aucun résultat concernant la durée de l'œstrus ne sera donné.

L'intervalle injection de l'hormone PMSG-cestrus (IC) est donné en heures. La normalité des courbes de distribution a été testée sclon le critère de $\chi^{2}$ de PEArson.

\section{RÉSULTATS}

Sur 1'ensemble des 385 vaches Française Frisonne Pie Noire et Charolaise, la proportion d'ovulations silencieuses n'a été que de 0,8 p. 10o. 382 animaux ont done servi pour les calculs rapportés ici.

\section{Distribution du jour d'injection de PMSG par rapport à l'cestrus précédent}

Les durées moyennes des cycles œstriens qui ont servi à calculer la date d'injection de PMSG varient de 18 à 24 jours. Ainsi le jour d'injection de PMSG rapporté à l'œstrus précédent considéré comme $\mathrm{J}_{0}$ a varié de $\mathrm{J}_{14}$ à $\mathrm{J}_{20}$ (fig. I) ; le mode se situe à $J_{18}$ pour 35 p. Ioo des vaches; pour 83 p. Ioo d'entre elles l'injection a été effectuée les $\mathrm{I} 5^{\mathrm{e}}, \mathrm{I} 6^{\mathrm{e}}$ et $\mathrm{I} 7^{\mathrm{e}}$ jours du cycle. Il en est de même pourr les animaux de race Charolaise : 31,5 p. Ioo ont été injectés le $16^{\mathrm{e}}$ jour du cycle et $74 \mathrm{p}$. Ioo les $15^{\mathrm{e}}$, $\mathrm{x} 6^{\mathrm{e}}$ et $\mathrm{I} 7^{\mathrm{e}}$ jours (fig. $\mathrm{I}$ ).

\section{Influence de la dose de PMSG sur l'intervalle $I C$}

L'injection de PMSG étant faite 4 jours avant la date présumée de l'œstrus, l'intervalle moyen IC devrait donc être égal à 96 heures. En réalité chez les vaches Frisonne pour la dose de I 600 UI de PMSG cet intervalle est de 94, I heures, puis il 


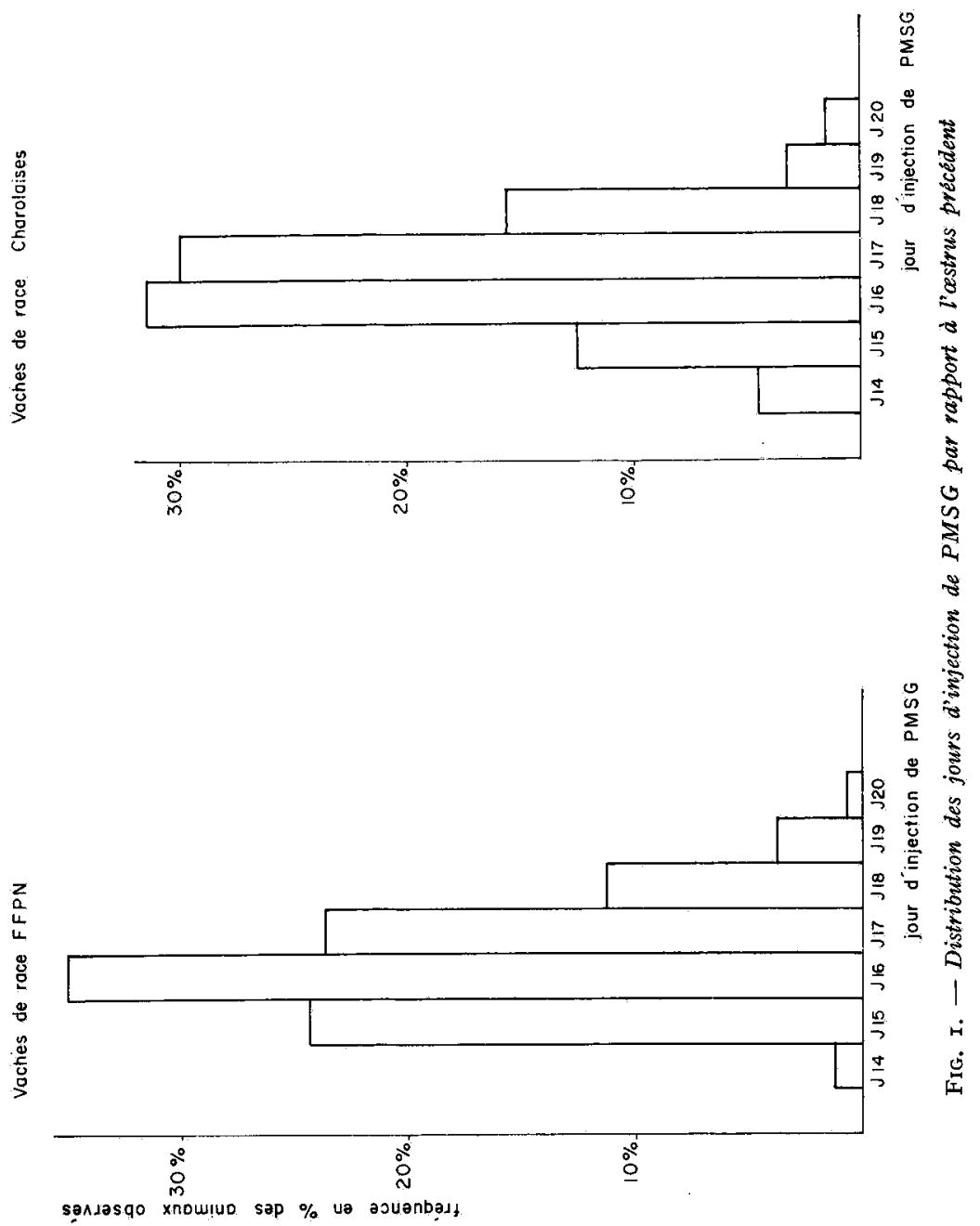


P. MAUl,ÉON, J.-C. MARIANA, D. ChUPIN, A. SOLARI

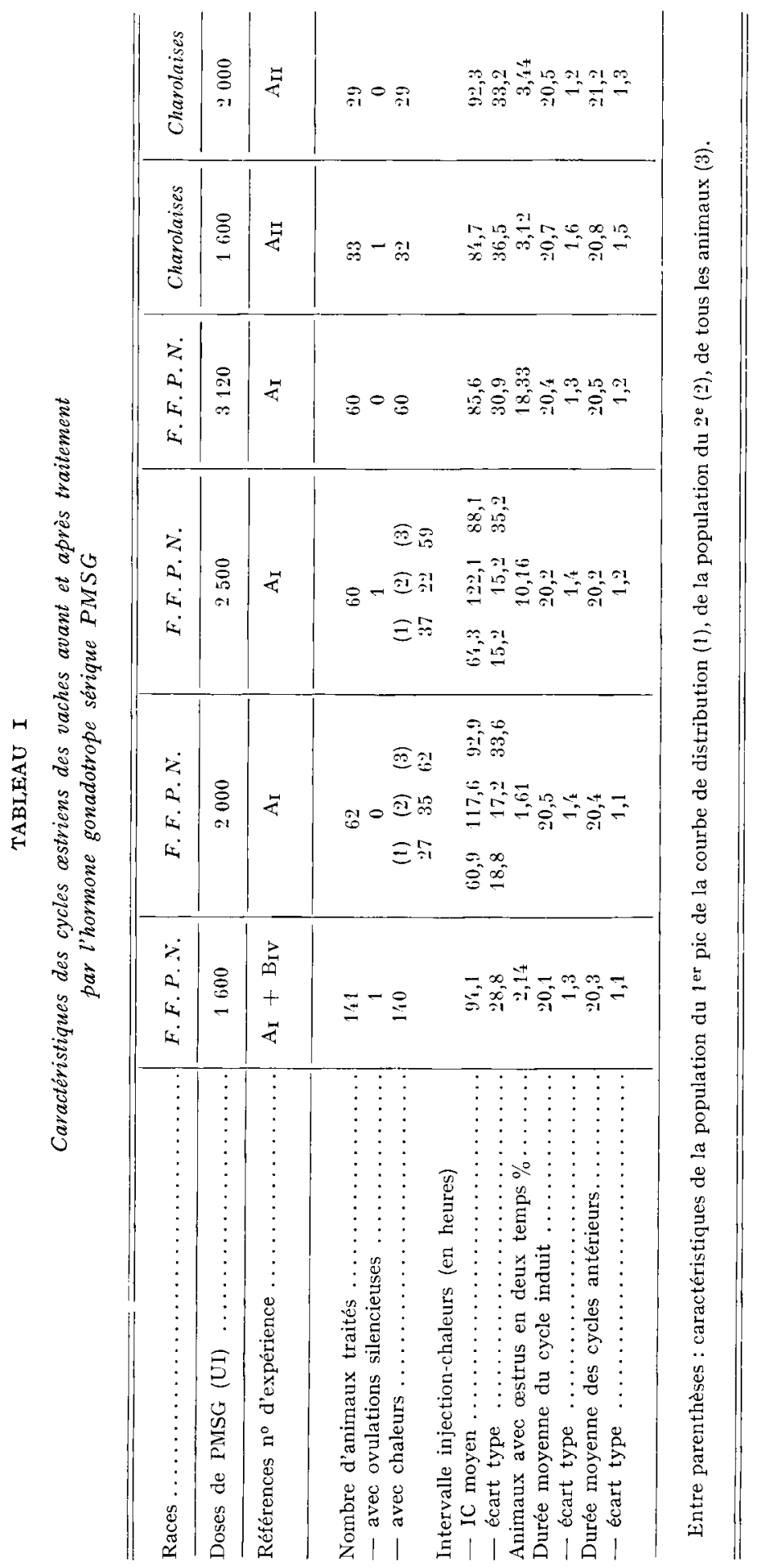


diminue légèrement d'environ 2 heures et 6 heures pour les doses 2 ooo et 2500 UI et finalement devient nettement plus court et égal à $85,6 \mathrm{~h}$ pour la dose élevée de $\mathrm{PMSG}$, 3 I20 UI (tabl. I).

Les courbes de distribution de cet intervalle subissent des modifications importantes en fonction de la dose de PMSG. Lorsque celle-ci est de I 600 UI la distribution est normale comme le montre l'histogramme des fréquences correspondant à des classes de I2 heures d'intervalle (fig. 2), la linéarité des droites de Henry correspon-
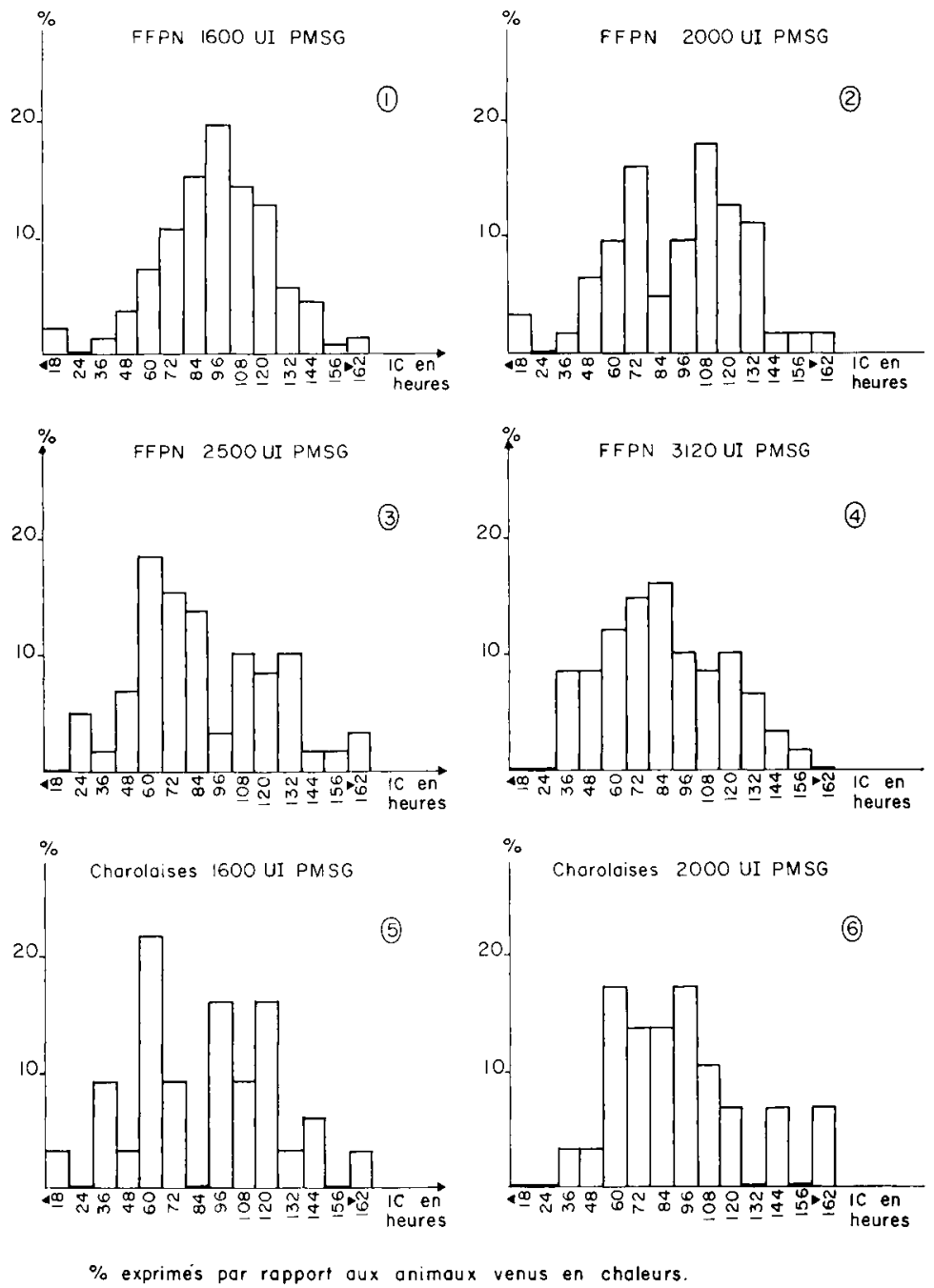

FTG. 2. - Distribution des intervalles IC pour différentes doses de PMSG

dantes et le test de normalité dı tablea1 2. Lorsque les doses de PMSG injectées sont égales à 2000 et 2500 UI les courbes de distribution des intervalles IC correspondants sont composées de 2 sous-populations; le clivage entre ces 2 sous-populations se produisant respectivement pour des valeurs de IC égales à 84 et 96 heures. Ces 
deux sous-populations sont distribuées normalement (tests de normalité du tableau 2 ) autour de valeurs moyennes de IC égales à $60 \mathrm{~h} 9$ et II $7 \mathrm{~h} 6$ pour la dose de 2 ooo UI de PMSG et $64 \mathrm{~h} 3$ et I22 h I pour la dose de 2500 UI de PMSG. Lorsque la plus forte dose de 3 I20 UI de PMSG est injectée, la courbe de distribution tend à redevenir normale et la valeur moyenne correspond à celle de l'une des sous-populations précédentes ; en fait à celle dont la valeur moyenne de IC était la plus faible.

\section{TABLEAU 2}

Tests de normalité des courbes de distribution des intervalles injection de PMSG-cestrus (IC) pour chaque dose de PMSG

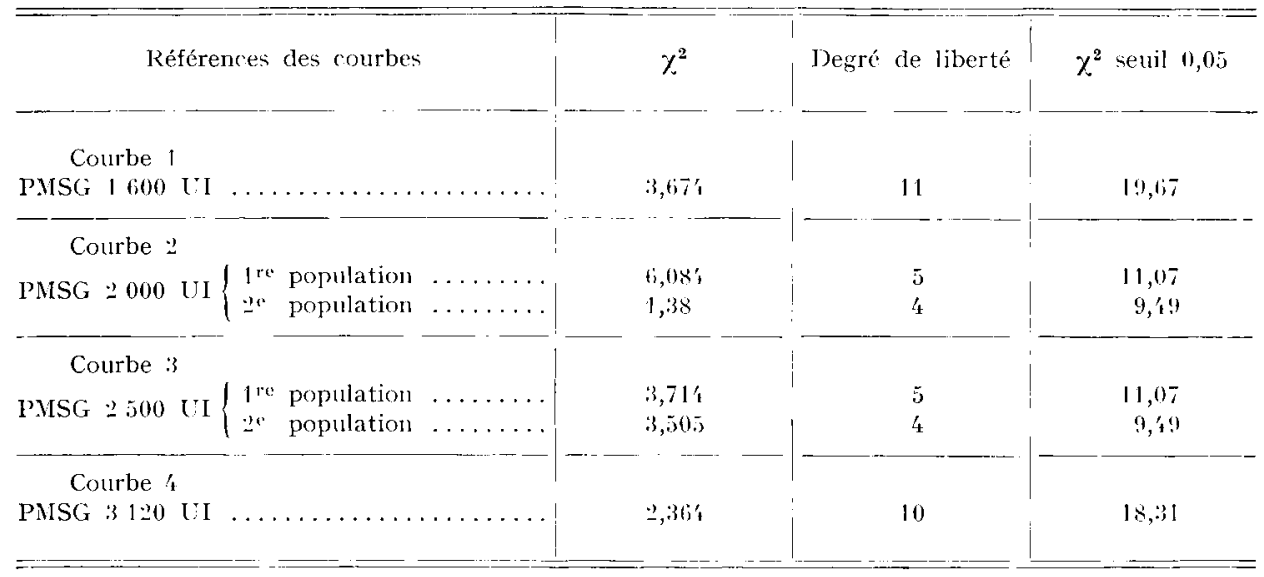

La courbe de distribution de 1'intervalle IC pour les vaches de race Charolaise après l'injection de I 600 UI de PMSG présente un clivage en deux sous-populations de part et d'autre de 84 heures comme cela existe après injection des doses 2000 et 2500 UI de PMSG aux animaux F.F.P.N. Après injection de 2 ooo UI aux vaches Charolaises on constate une tendance à un regroupement des intervalles IC en une population normale qui correspond à la sous-population dont l'intervalle $\overline{\mathrm{IC}}$ moyen est le plus court comme cela a été montré avec les vaches $F . F . P . N$. recevant 3 I 20 UI de PMSG.

Ces différences entre races dans l'effet dose de PMSG sur l'intervalle IC sont le reflet de la plus forte sensibilité ovarienne des animaux de race Charolaise à PMSG comme nous le montrerons dans un chapitre suivant.

En plus de cette modification de la distribution des intervalles moyens $\overline{\mathrm{IC}}$ avec la dose de PMSG injectée, on constate une augmentation de la fréquence des œestrus " partagés " (split œestrus) lorsque la dose de PMSG injectée augmente (tab1, I).

\section{Durée totale du cycle induit par rapport}

à la durée moyenne des cycles précédents

Seuls les animaux de race F.F.P.N. recevant I 600 UI de PMSG ont été considérés puisque c'est dans ce cas seulement que l'intervalle $\overline{\mathrm{IC}}$ moyen est voisin de l'intervalle souhaité de 96 heures et que la distribution de ces intervalles est normale. 
Il existe une corrélation positive significative $r=+0,4 \mathrm{I} 4$ entre la longueur du cycle induit et la durée moyenne des cycles antérieurs ce qui montre que la correction effectuée en fonction de la valeur individuelle estimée de la longueur du cycle oestrien est efficace.

Elle est toutefois meilleure pour les animaux ayant un cycle court que pour ceux ayant un cycle long comme le montre le tableau de correspondance entre la durée des cycles induits et celle que l'animal aurait dû avoir (tabl. 3 ).

TABLEAU 3

Efficacité de la correction du moment d'injection en fonction de la valeur individuelle de la durée du cycle cestrien

\begin{tabular}{|c|c|c|c|c|}
\hline $\begin{array}{l}\text { Intervalle wstrus précédent- } \\
\text { injection de l'MSG; }\end{array}$ & $\begin{array}{l}\text { Nombre } \\
\text { d'animaux }\end{array}$ & $\begin{array}{c}\overline{\mathrm{IC}} \\
\text { moyen }\end{array}$ & $\begin{array}{l}\text { Durée du cycle } \\
\text { induit }\end{array}$ & $\begin{array}{l}\text { Durée prévue } \\
\text { du cycle }\end{array}$ \\
\hline 1't jours........ & 1 & 1,0 & 18,0 & 18,0 \\
\hline 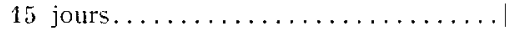 & $\underline{y}_{t}$ & 1,5 & 19,5 & 19,0 \\
\hline 16 jours $\ldots \ldots \ldots \ldots \ldots \ldots \ldots \ldots \ldots$ & 30 & 3,9 & 19,9 & $\geq 0,0$ \\
\hline 17 jours................... & 13 & 3,6 & $\because 0,6$ & $\because 1,0$ \\
\hline 18 jours. . . . . . . . . . . . . & 6 & 3,0 & $\geq 1,0$ & $2: 2,0$ \\
\hline 19 jours..................... & 1 & 2,0 & 21,0 & 23,0 \\
\hline 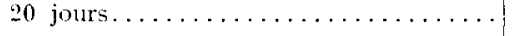 & 1 & 1,0 & 21,0 & 21,0 \\
\hline
\end{tabular}

I1 n'existe pas de corrélation significative $r=+0, \mathrm{I} 35$ entre la durée de deux cycles antérieurs, ce qui montre que la détermination de la correction du moment d'injection de PMSG ne peut se faire si l'on ne connaît que la durée d'un seul cycle.

\section{DISCUSSION}

Les ovulations silencieuses sont très rares lorsque l'injection de PMSG est faite dans les conditions de nos expériences.

L'imprécision des méthodes de détection d'ostrus, l'influence d'une injection trop précoce au cours d'un cycle oestrien mal connu peuvent expliquer les I4,4 p. IOO obtenus par Gordon, Wilifams, EDWARds (I962). L'injection d'hormone chorionique effectuée systématiquement après le $5^{\mathrm{e}}$ jour qui suit le traitement PMSG si la vache n'est pas encore venue en œstrus, est peut-être responsable des I8,9 p. Ioo d'ovulations silencieuses observées par SCANLON, SREENAN et GoRdON (I968).

Récemment au cours d'une autre expérience de naissances gémellaires tentée par Turman, Renbarger et Stephens (Ig69), il semble que plus de io p. Ioo des vaches ne soient pas venues en œestrus après l'injection d'hormone gonadotrope sérique bien que ces auteurs ne donnent aucune précision sur les ovulations silencieuses.

HAFEz et al. (1964) parlent également d'un taux élevé d'ovulations silencieuses ; cependant le calcul effectué à partir de leurs données ne révèle qu'une fréquence de 6 p. IOO, ce qui est plus faible que les résultats des auteurs précédents. HAFEz et al. 
(1965) trouvent d'ailleurs que la fréquence de ce phénomène n'est pas plus élevée après traitement à l'aide de PMSG que chez les animaux non traités.

Il nous semble que les variations enregistrées au cours deș diverses expériences sont surtout le reflet des conditions différentes d'environnement. En effet, on connaît l'influence de l'état physiologique de l'animal (GORDON, Williams, Edwards, I962), de la production laitière, de la saison, du niveau nutritionnel, de l'intervalle qui sépare le traitement du vêlage, de la race et même de la lignée génétique ( $\mathrm{L}_{\mathrm{ABH}} \mathrm{B}$ SETWAR, Tyler et CASIDA, I963). Mais il est possible également qu'il ne s'agisse, plus simplement, que des conditions différentes de détections d'œestrus. Compte tenu des résultats que nous avons obtenus, nous pensons qu'aucune influence del'injection de PMSG n'est démontrable sur la fréquence d'apparition des ovulations silencieuses.

Par contre, l'œestrus " partagé " est bien le fait du traitement PMSG et en particulier des doses élevées injectées comme l'ont noté d'autres auteurs (HAFEZ et SUGre, I96I; HAFEZ et al., I964; HAFEZ et al., I965).

L'influence de l'effet de la dose de PMSG sur la distribution de l'intervalle IC est dès le départ difficile à comparer entre auteurs puisque les hormones PMSG n'ont pas la même origine de fabrication et qu'une mauvaise correspondance entre unités internationales peut exister. D'autre part, les races bovines sur lesquelles les expériences ont été effectuées sont différentes et nous avons vu que ce facteur joue un rôle important. Un plus fort développement folliculaire entraîne une plus forte secrétion d'œstrogènes et le seuil de sensibilité du système nervetıx central à ce stéroïde risque d'être atteint plus tôt; il est donc normal que l'on assiste lorsque la dose de PMSG croît ou lorsque la sensibilité des animaux (race) est plus grande à un déplacement de la distribution vers des intervalles IC plus courts. C'est aussi la tendance observée par HAFEZ et al., (1965), du moins du point de vue effet global de PMSG ; par contre, aucun effet notable de la dose de PMSG n'a été observé sur la durée du cycle induit par GoRdon, Wilinams et EDWARDS (Ig62) dans les limites de 800 à 2000 UI. Chez les Ovins, RoBrsson (I95I) et WALLACE (I954) notent que le traitement PMSG pendant la phase folliculaire hâte la venue en ostrus, mais ne trouvent pas d'effet dose sur ce raccourcissement à l'inverse de ce qu'observe GoRdon (I958).

Enfin la tendance à une diminution de l'intervalle IC lorsque l'injection de PMSG est plus tardive par rapport à l'œstrus précédent, apparaît également dans les résultats de Turman et al. (1969).

\section{CONCLUSION}

Parmi les différentes doses utilisées dans nos expériences, seule la dose de I 600 UI de PMSG injectée 4 jours avant la date présumée de l'œestrus permet une distribution normale de l'intervalle injection-œstrus (IC) autour de la valeur souhaitée de 96 heures. Chez les vaches $F . F . P . N$., la durée du cycle induit est en corrélation avec la valeur moyenne de la longueur des cycles précédents et cette correction est particulièrement efficace pour les vaches à cycles courts. Dans l'ignorance de cette durée moyenne, l'injection de PMSG au $I 6^{\mathrm{e}}$ jour après la date de l'œstrus précédent semble être la meilleure pour la population des vaches $F . F . P . N$. Mais étant donné les différences obtenues avec les animaux de race Charolaise, cette date doit être 
réétudiée dans cette race en utilisant une dose de PMSG plus faible que celles expérimentées ici.

\section{RÉSUMÉ}

L'injection d'hormone gonadotrope sérique a été effectuée 4 jours avant la date présumée de l'œstrus, évaluée d'après la longueur des cycles œestriens antérieurs ; en fait, le jour d'injection s'est trouvé les $\mathrm{I}^{\mathrm{e}}$, $16^{\mathrm{e}}$ et $\mathrm{I} 7^{\mathrm{e}}$ jours du cycle dans 83 p. Ioo des cas pour les vaches de race F. F. P.N. et dans 74 p. Ioo des cas pour celles de race Charolaise.

Dans les conditions de détection d'œstrus utilisant un mâle vasectomisé, la proportion d'ovulation silencieuse est faible (0,8 p. I00). Elle est indépendante de la dose de PMSG injectée ; par contre, le pourcentage de " split oestrus " augmente avec cette dose.

La distribution des intervalles injection de PMSG-oestrus (IC) est modifiée pour les doses 2000,2500 et 3 I 20 UI de PMSG. La distribution de ces intervalles est normale lorsque la dose injectée est de I 600 UI de PMSG, mais cette population se clive en deux sous-populations normales autour des valeurs moyennes 60,9 et I 17,6 heures d'une part, 64,3 et I22, I heures d'autre part lorsque les doses injectées sont respectivement 2 ooo UI et 2500 UI de PMSG.

Lorsque la dose de PMSG est de 3 I 20 UI, la courbe de distribution des intervalles IC tend à devenir normale mais la moyenne de cet intervalle est de 85,6 heures, c'est à dire que IC devient plus court. C'est seulement pour une dose de I 600 UI de PMSG que le cycle au cours duquel a lieu le traitement est le moins modifié puisque l'intervalle moyen IC de 94 , $\mathrm{I}$ heures est alors très voisin de la valeur souhaitée de 96 heures.

Par ailleurs, la correction du moment d'injection effectuée en fonction de la valeur individuelle de la longueur du cycle ostrien est efficace puisqu'il existe une corrélation positive de $r=+0,4 \mathbf{I} 4$ entre la longueur du cycle induit et la durée des cycles antérieurs.

Il existe des différences entre races dans l'effet dose de PMSG sur l'intervalle IC; ainsi pour les vaches de race Charolaise après injection de $\mathrm{x} 600 \mathrm{UI}$, la courbe présente deux sous-populations de part et d'autre de 84 heures. Cette dose d'hormone produit sur les animaux de cette race un effet comparable à 2500 UI de PMSG chez ceux de race F.F.P.N.

\section{SUMMARY}

\section{ACTION ON THE DURATION OF THE COW EESTROUS CYCLE OF DIFFERENT PMSG DOSES INJECTED DURING THE FOLIICULAR PHASE}

PMSG was injected four days before the presumed date of aestrus, estimated from the length of previous westrus cycles. The day of injection fell on day I5, I6, and I 7 of the cycle in 83 p. roo of French Friesian cows, and on the same days in 74 p. Ioo of Charolais cows.

When œstrus was detected with a vasectomized bull, the proportion of silent ovulations was small (o.8 p. Ioo), being independent of the PMSG dose injected. On the contrary, the percentage of split oestrus increased with this dose in French Friesian cows ( $F$. F. P. N.).

The distribution of the intervals between PMSG injection and induced cstrus (IC) is modified for the PMSG doses of 2, 0oo, 2, 500, and 3 1 20 IU The distribution of these intervals is normal when the injected dose of PMSG is I $600 \mathrm{IU}$, but this population splits into two normal sub-populations, one around the mean values of 60.9 and I 7.6 hours, and the other around 64.3 and I22. I hours when the injected doses are 2 ooo and 2500 1. U. of PMSG, respectively.

When the PMSG dose is 3 I 20 IU, the curve of the IC intervals tends to become normal again, but the mean of this interval is 85.6 hours; that is, the I. C. becomes shorter.

The cycle during which the treatment is least modified occurs when the PMSG dose of I $600 \mathrm{IU}$ is given, since the mean IC interval of 94.1 hours is very close to the 96 -hour desired value

Furthermore, correction of the injection time in terms of the individual value of ostrous cycle length is efficacious since a positive correlation exists between the length of the induced cycle and the duration of previous cycles $\left(r=+0.4 \mathbf{I}_{4}\right)$.

Differences between breeds exist as concerns the PMSG dose effect on the IC interval. 
Thus, for the Charolais cows recciving an injection of $\mathrm{I}, 600 \mathrm{IU}$, the curve shows two sub-populations on each side of the 24 -hour area. This hormone dose, given to those animals, produced an effect comparable to that which 2500 IU of PMSG had on French Friesian cows.

\section{RÉFÉRENCES BIBLIOGRAPHIQUES}

Casida L. E., Nalbandov A., McShan W. H., Meyler R, K., Wisnichy W., ig4o. Potential fertility of artificially matured and ovulated ova in cattle. Proc. am. Soc. Anim. Prod., 33, 302-303.

GoRDon I., I958. The use of progesterone and serum gonadotrophin (PMSG) in the control of fertility in sheep. J.agric. Sci., 50, 123-I97.

Gordon I., Williams G., Edwards J., rg62. The use of serum gonadotrophin (PMSG) in the induction of twin-pregnancy in the cow. J. Agric. Sci., 59, I43-199.

HaFEz E.S. E., Sugie T., I961. Superovulatory responses in beef cattle and an experimental approach for non-surgical ova transfer. Proc. IVth Int. Cong. Physiol. Anim. Reprod., The Hague, 2, 387-39I.

Hafez L. S. L., Rajakoski E., Andirson P. B., lirost O. L., Smith G., I964. Problems of gonadotropin induced multiple pregnancy in beef cattle. Am. J. I'et. Res., 25, 1074-1079.

Hafez IE. S. E., Jainudeen M. R., Lindsay 1). R., i965. Gonadotrophin induced twinning and related phenomena in beef cattle, Acta endocr., 50, suppl. I02, I-44.

Hamiond J., Bhattacharya I', I944. Control of ovulation in the cow. J. Agric. Sci., 34, I-I5.

Labhisetwar A. P., 'Tyler W. J, Casida L. H., I963. Genetic and environmental factors affecting quiet ovulations in cattle. J. Dairy Sci., 46, $843-845$.

Lamung G. li., Rowson L. E., 1952. Superovulation and ovum transplantation in cattle. 2nd Cong Artif. Insem. Physiol. Reprod., Copenhagen, I44-153.

Olds O., Seati P. M., I95I. Repeatability of estrous cycle length in dairy cattle. J. Dairy Sci., 34, 626-628.

Robinson T. J., I95I. The control of fertility in sheep. Part II. - The augmentation of fertility by gonadotrophin treatment of ewe in the normal brceding season. J. Agric. Sci, 41, 6-63.

Rowson L. E., I95r. Methods of inducing multiple ovulations in cattle. J. Endocr., 7, 260-270.

Scanlon P., SReenan J., Gordon I., I968. Hormonal induction of superovulation in cattle. $J$. Agric. Sci., 70, $179-182$.

Turmax E. J., Renbarger R. E., Stepinens D. F., I969. I'roducing multiple births in beef cows by hormone injections (non publié).

WALLACE L. R., I954. Studies in the augmentation of fertility of Romney ewes with pregnant-mare serum, J. Agric. Sci., 45, 60-79.

Willett E. L., McShan W. H., Meyer R. K., I952. Cronadotrophins for superovulation of cows. Proc. Soc. exp. Biol., 79, 396-400.

Zavadovski M. M., Eskin I. A., Ovsjannikov G. T., I935. The control of the female sex cycle in cows. Trud. Dinam. Razuit, 9, 75 . 